\title{
Natural convection heat transfer in a square cavity heated by trapezoidal body using two-phase nanofluid model
}

\author{
Ali J. Chamkha*, Ammar I. Alsabery and Ishak Hashim
}

\begin{abstract}
Steady laminar natural convection and heat transfer of nanofluid-filled square cavity heated by trapezoidal solid body is studied numerically using the finite element method. The heat source into the cavity is considered by the bottom wall of the trapezoidal body, where this segment is kept at a higher isothermal temperature. The left and right horizontal walls are maintained isothermally with cold temperature while the left and right horizontal walls is thermally insulated. The boundaries of the domain are assumed to be impermeable, the fluid within the cavity is a water-based nanofluid having $\mathrm{Al}_{2} \mathrm{O}_{3}$ nanoparticles. The numerical computations are obtained for various parameters of Rayleigh number, nanoparticle volume fraction and thermal conductivity of the trapezoidal body. The heat transfer rate is clearly enhanced with the increasing of the nanoparticle volume fraction and Rayleigh number. However, this enhancement tends to be quite obvious at the interface wall.
\end{abstract}

Keywords - heat transfer, square cavity, solid trapezoidal body, Thermophoresis, Brownian, two-phase nanofluid model

\section{Introduction}

Natural convective heat transfer inside closed cavities is considered to be an important phenomenon in wide range of engineering applications, such as, electronic cooling, cooling of containment buildings, room ventilation, heat exchangers, storage tanks, double pane windows, solar collectors, etc. The low thermal conductivity of the pure fluids, such as, water, ethylene glycol, and oil show a limitation for the enhancement of the heat transfer. To avoid this limitation, the nanoparticles are submerged into the pure fluid which tend to change the thermophysical property of this fluid and therefore, the heat transfer rate is clearly enhanced.

\section{Ali J. Chamkha}

Department of Mechanical Engineering, Prince Sultan Endowment for Energy and the Environment, Prince Mohammad Bin Fahd University Saudi Arabia

\section{Ammar I. Alsabery}

Refrigeration \& Air-conditioning Technical Engineering Department, College of Technical Engineering, The Islamic University Iraq

\section{Ishak Hashim}

School of Mathematical Sciences, Faculty of Science \& Technology, Universiti Kebangsaan Malaysia Malaysia
The used of nanofluids can be seen in many engineering applications, for example, cooling of electronics, heat exchangers, engine cooling, nuclear reactor safety, hyperthermia, biomedicine, vehicle thermal management, and many others [1]. Natural convection and heat transfer problems of CuO-EG-water nanofluid in a square cavity are considered by Abu-Nada and Chamkha [2]. They found that the viscosity model effects were more dominant on the average Nusselt number compare to the thermal conductivity model effects. Basak and Chamkha [3] numerically investigated the heatline analysis on convective heat transfer of nanofluid in a square cavity with various thermal boundary conditions. Sheremet et al. [4] studied the entropy generation and natural convection heat transfer of nanofluid within square cavity containing a hot solid block.

Alsabery et al. [5] used the finite difference method to investigate the transient natural convection and heat transfer in a trapezoidal cavity filled with nanofluid in the presence of spatial side-wall temperature. Recently, Alsabery et al. [6] reported a numerical investigation on the effect of two phase nanofluid model of natural convective heat transfer in a square cavity filled with nanofluid and containing an inner solid body. According to above mentioned studies and to the authors' best knowledge, so far, there have been no studies of natural convection heat transfer in a square cavity heated by trapezoidal body. Thus, we believe that this work is valuable. The aim of this study is to investigate the natural convection heat transfer in a square cavity heated by trapezoidal body using the two-phase nanofluid model.

\section{Mathematical Formulation}

The steady two-dimensional natural convection problem in a square cavity with length $L$ and with the presence of a bottom trapezoidal body with a bottom base of 0.5 , a top base of 0.25 and height $h$ as illustrated in Fig. 1. The Rayleigh number range chosen in the study keeps the nanofluid flow incompressible and laminar. The heat source into the cavity is considered at the bottom base of the trapezoidal body where this segment is kept at a higher isothermal temperature, $T_{h}$. The vertical walls are maintained isothermally with a cold temperature, $T_{c}$, while the top horizontal wall and the remaining parts of the bottom wall are kept adiabatic. The boundaries of the annulus are assumed to be impermeable, the fluid within the cavity is a water-based nanofluid having $\mathrm{Al}_{2} \mathrm{O}_{3}$ nanoparticles. The Boussinesq approximation is applicable. By considering these assumptions, the continuity, momentum and energy equations can be shown in the dimensionless form as follows [6]: 


$$
\begin{gathered}
\nabla \cdot \mathbf{V}=0, \\
\mathbf{V} \cdot \nabla \mathbf{V}=-\nabla P+\frac{\rho_{f}}{\rho_{n f}} \frac{\mu_{n f}}{\mu_{f}} \nabla^{2} \mathbf{V}+\frac{(\rho \beta)_{n f}}{\rho_{n f} \beta_{f}} \frac{1}{\operatorname{Pr}} R a \cdot \theta, \\
\mathbf{V} \cdot \nabla \theta=\frac{\left(\rho C_{p}\right)_{f}}{\left(\rho C_{p}\right)_{n f}} \frac{k_{n f}}{k_{f}} \frac{1}{\operatorname{Pr}} \nabla^{2} \theta+\frac{\left(\rho C_{p}\right)_{f}}{\left(\rho C_{p}\right)_{n f}} \frac{D_{B}^{*}}{\operatorname{Pr} \cdot L e} \nabla \varphi^{*} \cdot \nabla \theta \\
+\frac{\left(\rho C_{p}\right)_{f}}{\left(\rho C_{p}\right)_{n f}} \frac{D_{T}^{*}}{\operatorname{Pr} \cdot L e \cdot N_{B T}} \frac{\nabla \theta \cdot \nabla \theta}{1+\delta \theta}, \\
\mathbf{V} \cdot \nabla \varphi^{*}=\frac{D_{B}^{*}}{S c} \nabla^{2} \varphi^{*}+\frac{D_{T}^{*}}{S C \cdot N_{B T}} \cdot \frac{\nabla^{2} \theta}{1+\delta \theta}, \\
\nabla \theta_{w}=0,
\end{gathered}
$$

where $\mathbf{V}$ shows the dimensionless velocity vector $(U, V)$.

The governing equations of Navier Stokes equations (1)-(5) are transformed into dimensionless forms using the following dimensionless variables:

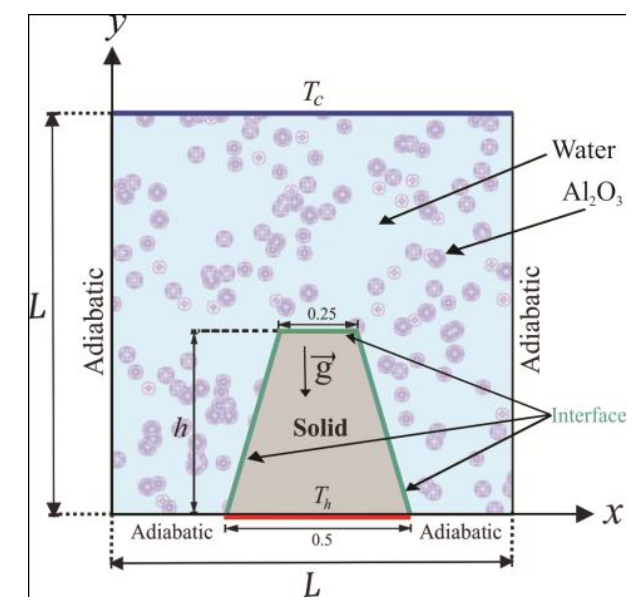

Figure 1. Physical model of convection in a square cavity together with the coordinate system

$$
\begin{gathered}
X=\frac{x}{L}, \quad Y=\frac{y}{L}, \quad \mathbf{V}=\frac{\mathbf{v} L}{v_{f}}, \quad \theta=\frac{T-T_{c}}{T_{h}-T_{c}}, \\
\theta_{s}=\frac{T_{s}-T_{c}}{T_{h}-T_{c}}, \quad h=\frac{H}{L}, \quad D=\frac{d}{L}, \operatorname{Pr}=\frac{v_{f}}{\alpha_{f}}, \\
R a=\frac{g \beta_{f}\left(T_{h}-T_{c}\right) L^{3}}{v_{f} \alpha_{f}}, P=\frac{p L^{2}}{\rho_{f} \alpha_{f}^{2}} .
\end{gathered}
$$

The nanofluid properties are adapted from our previous published work in [6]. The dimensionless boundary conditions corresponding to Eqs. (1)- (5) are given by:

On the heated part of the horizontal bottom wall :

$$
U=V=0, \frac{\partial \varphi^{*}}{\partial n}=-\frac{D_{T}^{*}}{D_{B}^{*}} \frac{1}{N_{B T}} \frac{1}{1+\delta \theta} \frac{\partial \theta}{\partial n}, \theta=1
$$

On the adiabatic parts of the remainder bottom wall:

$U=V=0, \frac{\partial \varphi^{*}}{\partial n}=0, \frac{\partial \theta}{\partial n}=0$,
On the cold left and right vertical walls :

$$
U=V=0, \frac{\partial \varphi^{*}}{\partial n}=-\frac{D_{T}^{*}}{D_{B}^{*}} \frac{1}{N_{B T}} \frac{1}{1+\delta \theta} \frac{\partial \theta}{\partial n}, \theta=0,
$$

On the horizontal adiabatic top wall:

$U=0, V=0, \frac{\partial \varphi^{*}}{\partial n}=0, \frac{\partial \theta}{\partial n}=0$

On the walls of the trapezoidal solid body:

$\theta=\theta_{w}$,

$$
U=V=0, \frac{\partial \varphi^{*}}{\partial n}=-\frac{D_{T}^{*}}{D_{B}^{*}} \frac{1}{N_{B T}} \frac{1}{1+\delta \theta} \frac{\partial \theta}{\partial n}, \frac{\partial \theta}{\partial n}=K_{r} \frac{\partial \theta_{w}}{\partial n},
$$

where $K_{r}=\frac{k_{w}}{k_{n f}}$ is the thermal conductivity ratio. The local and average Nusselt numbers evaluated at the heated part of the bottom wall are defined as, respectively,

$$
N u_{n f}=-\left(\frac{\partial \theta_{w}}{\partial Y}\right)_{Y=0}, \quad \overline{N u}_{n f}=\int_{\frac{1-0.5}{2}}^{\frac{1+0.5}{2}} N u_{n f} \mathrm{~d} X .
$$

Also, the local and average Nusselt numbers evaluated the interface, are defined as, respectively,

$$
N u_{i}=-\frac{k_{n f}}{k_{f}}\left(\frac{\partial \theta}{\partial n}\right)_{i}, \quad \overline{N u}_{i}=\int_{0}^{n} N u_{i} \mathrm{~d} n .
$$

The dimensionless governing equations (1)-(5) subject to the boundary conditions (7)-(12) are solved using the Galerkin weighted residual finite element method where the computational domain is discretized into triangular elements.

To assure the validity of the present code, validations against experimental and numerical work of Calcagni et al. [7] are made. Fig. 2 shows results for the case of natural convective heat transfer in a square cavity heated from below. Our streamlines and isotherms show a very good agreement with the experimental and numerical results of Calcagni et al. [7]. 
Proc. of the Seventh Intl. Conf. on Advances in Civil, Structural and Mechanical Engineering - CSM 2018 Copyright (C) Institute of Research Engineers and Doctors, USA .All rights reserved.

ISBN: 978-1-63248-163-4 DOI: 10.15224/978-1-63248-163-4-19
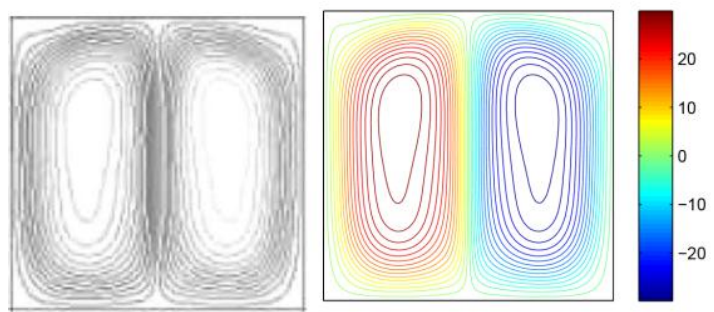

(a)
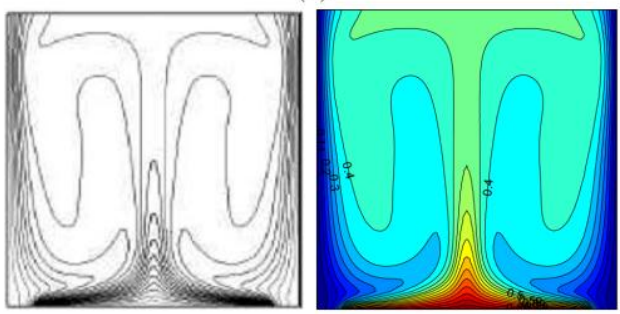

(b)
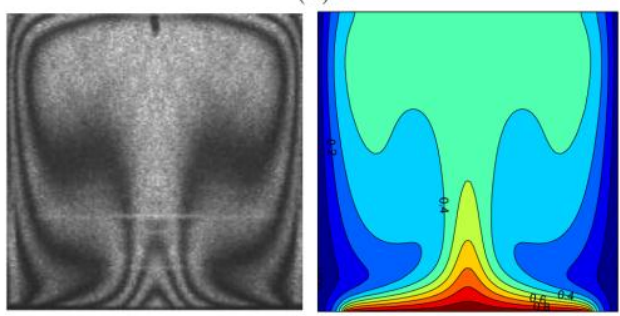

(c)

Figure 2. (left) Calcagni et al. [7] and (right) present study for (a) streamlines at $R a=10^{6}$, (b) isotherms at $R a=10^{5}$ and (c) isotherms at $R a=1.836 \times 10^{5}$ for the case of numerical and experimental results of Calcagni et al. [7] at $\phi=0$ and $H=0$.

\section{Results and Discussion}

This section presents numerical results of the streamlines, isotherms, nanoparticle distribution, and average Nusselt number with various values of the Rayleigh number $\left(10^{3} \leq R a \leq 10^{6}\right)$, nanoparticle volume fraction $(0 \leq \phi \leq 0.04)$ and thermal conductivity of the trapezoidal body $\left(0.5 \leq k_{w} \leq 16\right)$. The thermophysical properties of pure fluid and solid $\mathrm{Al}_{2} \mathrm{O}_{3}$ phases are shown in Table 1 .

TABLE I. THERMOPHYSICAL PROPERTIES OF WATER WITH $\mathrm{AL}_{2} \mathrm{O}_{3}$ NANOPARTICLES AT $T=310 \mathrm{~K}$

\begin{tabular}{|c|c|c|}
\hline Physical properties & $\begin{array}{c}\text { Fluid phase } \\
\text { (water) }\end{array}$ & $\boldsymbol{A l}_{\mathbf{2}} \boldsymbol{O}_{\mathbf{3}}$ \\
\hline$C_{p}(\mathrm{~J} / \mathrm{kgK})$ & 4178 & 765 \\
\hline$\rho\left(\mathrm{kg} / \mathrm{m}^{3}\right)$ & 993 & 3970 \\
\hline$k\left(\mathrm{Wm}^{-1} \mathrm{~K}^{-1}\right)$ & 0.628 & 40 \\
\hline$\beta \times 10^{5}(1 / \mathrm{K})$ & 36.2 & 0.85 \\
\hline$\mu \times 10^{6}(\mathrm{~kg} / \mathrm{ms})$ & 695 & - \\
\hline$d_{p}(\mathrm{~nm})$ & 0.385 & 33 \\
\hline
\end{tabular}

Figure 3 illustrates the effects of various values of Rayleigh number on the streamlines, isotherms and nanoparticle distribution for $\phi=0.02$ and $k_{w}=0.76$. At low Rayleigh number ( $R a=10^{3}$ ), the flow within the square cavity is characterized by two symmetric streamlines cells at the upper segment, one in clockwise direction appear

close to the left wall while the other cell in the anticlockwise direction located next to the right wall. This because of the heating system applied in this problem. The isotherm patterns appear with very low density next to the cold wall, the isotherm patterns tend to take almost a horizontal lines. This due to the low thermal gradient. The nanoparticle distribution contours show that the heat transfer via conduction becomes dominant and thermophoresis displays a strong influence on the distribution of nanoparticles inside the cavity. Thus, the nanoparticles showcase non-uniform dispersion within the cavity, along with an essential increase in the nanoparticles concentration near the trapezoidal body walls.

The increasing of Rayleigh number tends to increase the intensity of the streamlines and the size of the cells due to the buoyancy forces are more strong compare to the viscous forces. The intensity of the isotherm patterns increases and occupied more space inside the cavity. The nanoparticles distribution changes uniform due to the strength of the buoyancy effects that move more nanoparticles at the recirculation zones, which cause less deposition of nanoparticles. Thus, the single-phase homogeneous approach can be used for this stage, as described in Fig. 3(d).
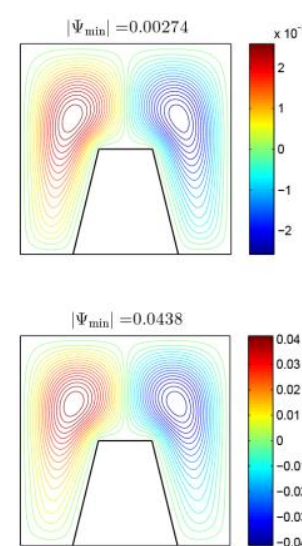

$\left|\Psi_{\min }\right|=1.7$

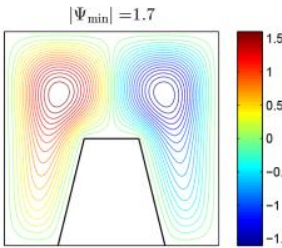

$\left|\Psi_{\min }\right|=6.51$
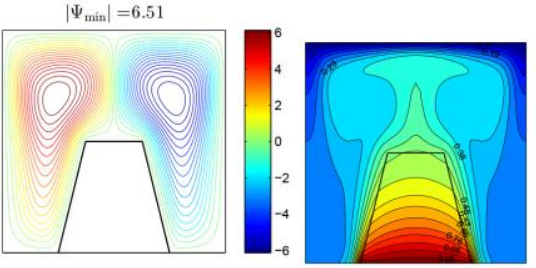

(d) $R a=10^{6}$

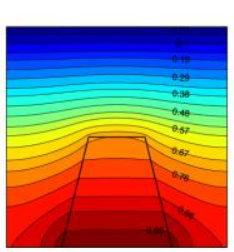

(a) $R a=10^{3}$

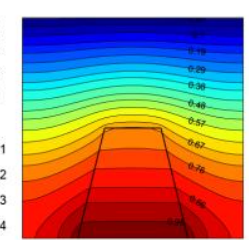

(b) $R a=10^{4}$

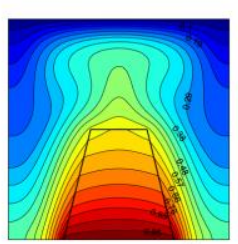

(c) $R a=10^{5}$
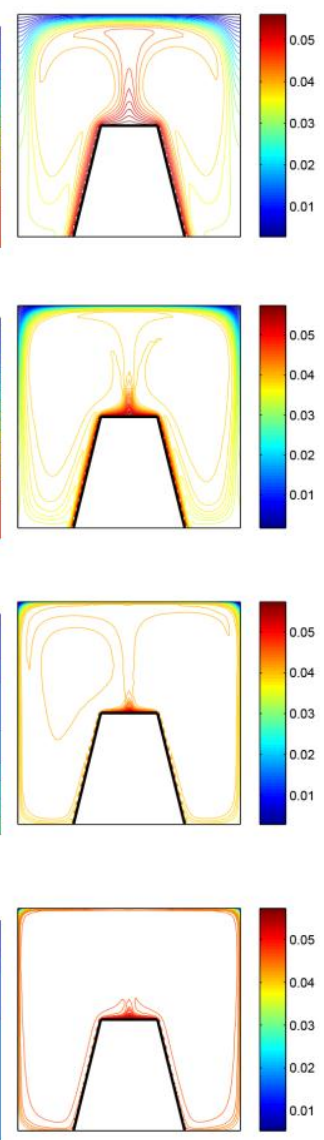

Figure 3. Variations of the streamlines (left), isotherms (middle), and nanoparticle distribution (right) by the Rayleigh number $(R a)$ for $\phi=0.02$ and $k_{w}=0.76$

Figure 4 presents the effects of various values of the thermal conductivity of the trapezoidal body on the streamlines, isotherms and nanoparticle distribution for $R a=10^{3}$ and $\phi=0.02$. It has been observed from this 
figure that the increment of the thermal conductivity of the trapezoidal body tends to increase the intensity and the size of the streamlines cells. As a results, the strength of the flow circulation increases.

The isotherm patterns observe with a low density within trapezoidal solid body. This is due to the reduction of the thermal resistance, which transfers more heat within the cavity by the increasing thermal conductivity. The concentration of nanoparticles moves around the walls of the cavity which clearly shows the enhancement of the thermophoresis effects.

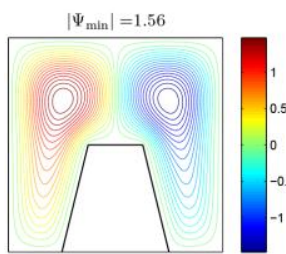

$\left|\Psi_{\min }\right|=1.98$

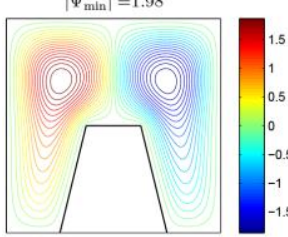

$\left|\Psi_{\min }\right|=2.2$

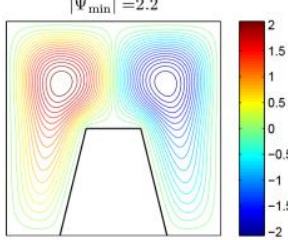

$\left|\Psi_{\min }\right|=2.27$
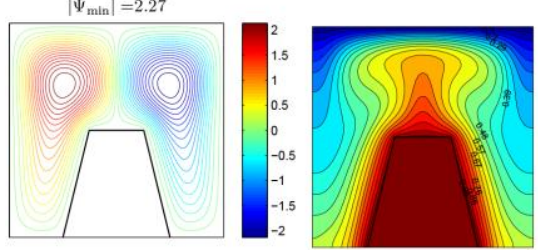

(d) $k_{w}=16$

(a) $k_{w}=0.5$

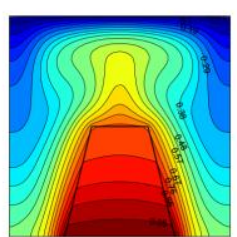

(b) $k_{w}=1.95$

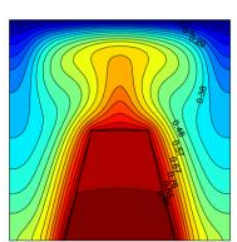

(c) $k_{w}=7$
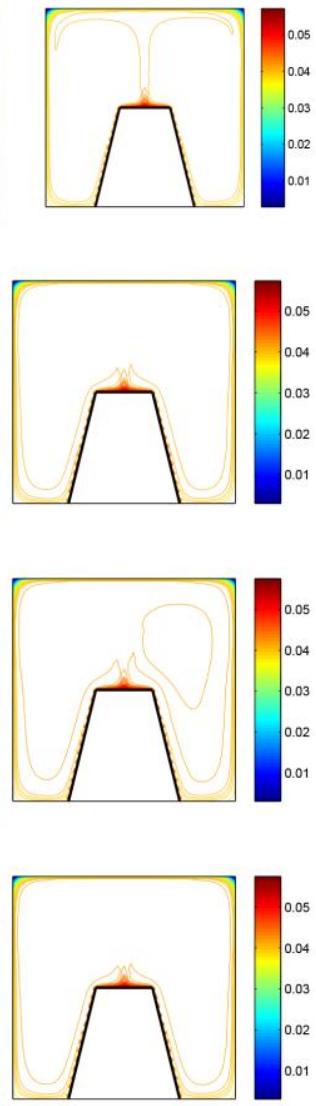

Figure 4. Variations of the streamlines (left), isotherms (middle), and nanoparticle distribution (right) by the thermal conductivity of the trapezoidal body $\left(k_{w}\right)$ for $R a=10^{5}$ and $\phi=0.02$.

Figure 5 demonstrates the mean value of the average Nusselt number with nanoparticles volume fraction at each Rayleigh number separately. At a low Rayleigh number, the Nusselt number on the bottom wall and the interface wall continually increase with an increment in the nanoparticles volume fraction, while at $R a=10^{5}$, an optimum concentration of nanoparticles is observed for the maximum mean value of the Nusselt number. This is behaviour tends to be clear at the interface wall due to the enhancement of both the fluid viscosity and the fluid thermal conductivity. The combination between these dual effects optimizes the concentration of the nanoparticle for a maximized rate of heat transfer. Furthermore, when the Rayleigh number is increased, the maximized average Nusselt number values move to higher values of the nanoparticles volume fraction.
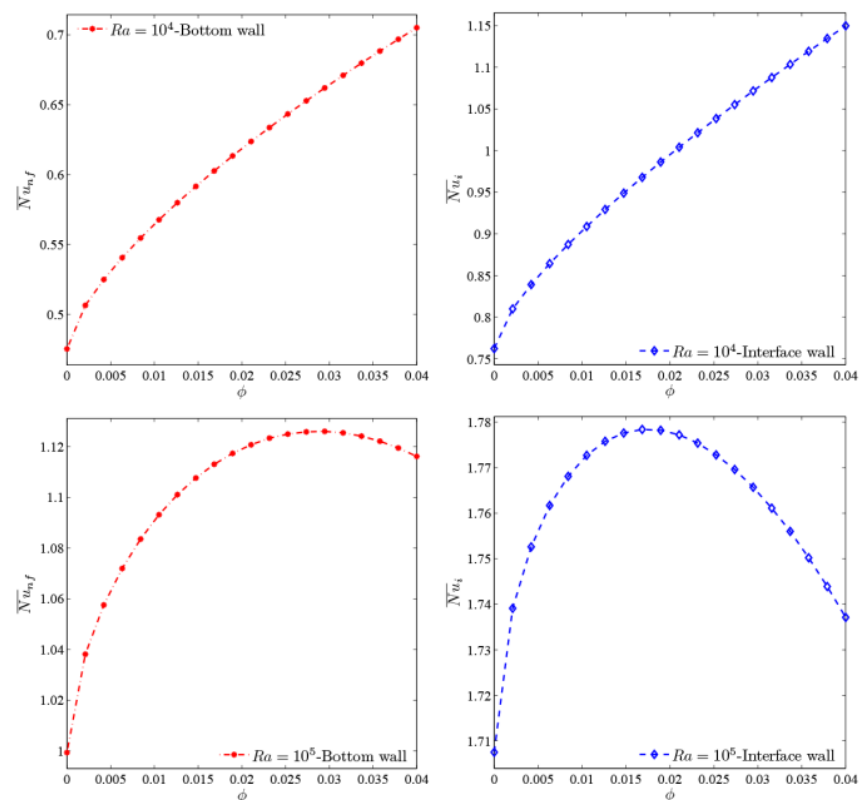

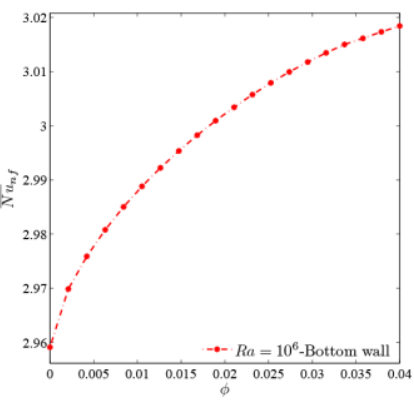

(a) Bottom wall

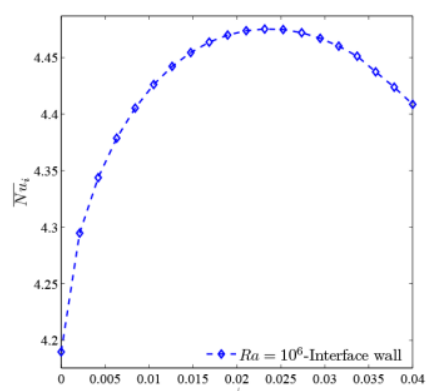

(b) Interface wall
Figure 5. Variations of the average Nusselt number on (a) bottom wall and (b) interface wall with $\phi$ for different $R a$ at $k_{w}=0.76$.

Figure 6 describes the influence of the nanoparticles volume fraction on the average Nusselt number with Rayleigh number. The convection heat transfer increases with the augmentation of $\phi$ particularly at the low Rayleigh number $\left(R a \leq 10^{4}\right)$. However. an optimum concentration of nanoparticles is observed for the maximum mean value of the average Nusselt number for relatively high Rayleigh number. While by increasing Rayleigh number up to the high values, the best enhancement obtained on the convection heat transfer with the higher values of the nanoparticles volume fraction.

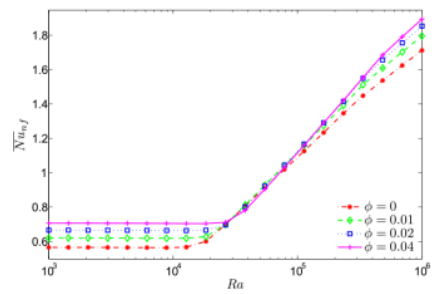

(a)

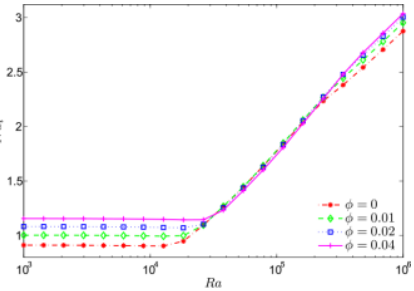

(b)
Figure 6. Variations of the average Nusselt number on (a) bottom wall and (b) interface wall with $R a$ for different $\phi$ at $k_{w}=0.76$. 
The effects of the thermal conductivity of the trapezoidal body on the average Nusselt number with nanoparticles volume fraction are shown in Figure 7. The convection heat transfer enhances with the addition of the nanoparticles volume fraction affected by an increment of the thermal conductivity. However, different behaviour observed on the average Nusselt number with an increasing of the thermal conductivity of the trapezoidal solid body. At the bottom wall, the convection shows a clear reduction with the growing of the thermal conductivity of the trapezoidal solid body, while an enhancement on the heat transfer is obtained with the higher $k_{w}$ on the interface wall. This happen due to the reduction of the thermal resistant of the solid body which allows more heat to transfer within the considered cavity.

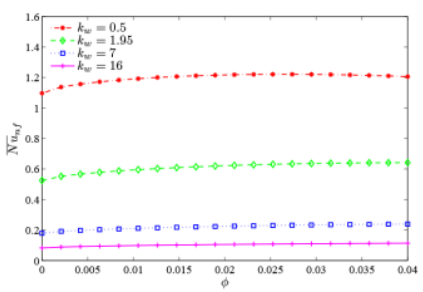

(a)

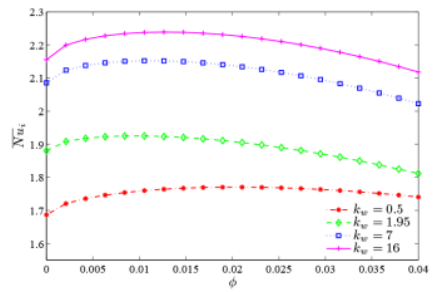

(b)
Figure 7. Variations of the average Nusselt number with $\phi$ for different $k_{w}$ at $R a=10^{5}$.

\section{Conclusions}

The present work investigated the natural convection heat transfer in a square cavity filled with $\mathrm{Al}_{2} \mathrm{O}_{3}$-water nanofluid and heated by trapezoidal body. By using Buongiorno's two-phase model, the slip between the base fluid and the nanoparticles was taken into consideration. The governing equations were made dimensionless and then solved using the Galerkin weighted residual finite element method. Numerical results are presented graphically in the form of streamlines, isotherms and nanoparticles volume fraction as well as the average Nusselt number. The numerical computations are obtained for various parameters of Rayleigh number, nanoparticle volume fraction and thermal conductivity of the trapezoidal body. The heat transfer rate is clearly enhanced with the increasing of the nanoparticle volume fraction and Rayleigh number. However, this enhancement tends to be quite obvious at the interface wall.

\section{Acknowledgment}

The work was supported by the UKM research grant DIP-2014-015.

\section{References}

[1] A. Shenoy, M. Sheremet, and I. Pop, Convective Flow and Heat Transfer from Wavy Surfaces: Viscous Fluids, Porous Media, and Nanofluids. CRC Press, 2016.

[2] E. Abu-Nada and A. J. Chamkha, "Effect of nanofluid variable properties on natural convection in enclosures filled with a cuo-egwater nanofluid," International Journal of Thermal Sciences, vol. 49, no. 12, pp. 2339-2352, 2010.

[3] T. Basak and A. J. Chamkha, "Heatline analysis on natural convection for nanofluids confined within square cavities with various thermal boundary conditions," International Journal of Heat and Mass Transfer, vol. 55, no. 21-22, pp. 5526-5543, 2012.

[4] M. A. Sheremet, H. F. Oztop, I. Pop, and N. Abu-Hamdeh, "Analysis of entropy generation in natural convection of nanofluid inside a square cavity having hot solid block: Tiwari and das model," Entropy, vol. 18, no. 1, p. 9, 2015.

[5] A. I. Alsabery, I. Hashim, A. J. Chamkha, H. Saleh, and B. Chanane, "Effect of spatial side-wall temperature variation on transient natural convection of a nanofluid in a trapezoidal cavity," International Journal of Numerical Methods for Heat \& Fluid Flow, vol. 27, no. 6, pp. 1365-1384, 2017.

[6] A. I. Alsabery, M. A. Sheremet, A. J. Chamkha, and I. Hashim, "Conjugate natural convection of al2o3-water nanofluid in a square cavity with a concentric solid insert using buongiornos two-phase model," International Journal of Mechanical Sciences, vol. 136, pp. 200-219, 2018.

[7] B. Calcagni, F. Marsili, and M. Paroncini, "Natural convective heat transfer in square enclosures heated from below," Applied Thermal Engineering, vol. 25, no. 16, pp. 2522-2531, 2005. 